\title{
Nanoscale
}

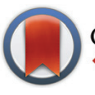

CrossMark $\leftarrow$ click for updates

Cite this: Nanoscale, 2016, 8, 13488

\section{Carbon nanotubes dispersed in aqueous solution by ruthenium(॥) polypyridyl complexes $\uparrow$}

\author{
Kewei Huang, $\ddagger^{\mathrm{a}}$ Avishek Saha, $\$^{\mathrm{a}}$, Konstantin Dirian, ${ }^{\mathrm{b}}$ Chengmin Jiang, ${ }^{a}$ \\ Pin-Lei E. Chu, ${ }^{a}$ James M. Tour, ${ }^{a, c}$ Dirk M. Guldi ${ }^{b}$ and Angel A. Martíta,c,d
}

Cationic ruthenium(॥) polypyridyl complexes with appended pyrene groups have been synthesized and used to disperse single-walled carbon nanotubes (SWCNT) in aqueous solutions. To this end, planar pyrene groups enable association by means of $\pi$-stacking onto carbon nanotubes and, in turn, the attachment of the cationic ruthenium complexes. Importantly, the ionic nature of the ruthenium complexes allows the formation of stable dispersions featuring individualized SWCNTs in water as confirmed in a number of spectroscopic and microscopic assays. In addition, steady-state photoluminescence spectroscopy was used to probe the excited state interactions between the ruthenium complexes and SWCNTs. These studies show that the photoluminescence of both, that is, of the ruthenium complexes and of SWCNTs, are quenched when they interact with each other. Pump-probe transient absorption experiments were performed to shed light onto the nature of the photoluminescence quenching, showing carbon nanotube-based bands with picosecond lifetimes, but no new bands which could be unambigously assigned to photoinduced charge transfer process. Thus, from the spectroscopic data, we conclude that quenching of the photoluminescence of the ruthenium complexes is due to energy transfer to proximal SWCNTs.

Received 28th March 2016. Accepted 29th May 2016

DOI: $10.1039 / c 6 n r 02577 c$

www.rsc.org/nanoscale thesized SWCNTs form bundles due to strong van der Waals attractions between them. Bulk bundled SWCNTs require individualization in order to fully exploit their mechanical, electronic, and optical properties. ${ }^{13-16}$ A general approach to debundle SWCNTs is to introduce repulsive interactions between them to counterbalance short-range attractions. To date, many approaches have been developed to individualize carbon nanotubes and most of them are simply divided into two categories, that is, covalent functionalization, ${ }^{17,18}$ versus non-covalent functionalization. ${ }^{16,19,20}$

On the one hand, covalent functionalization is an effective approach for attaching functional groups to the sidewalls of carbon nanotubes. Although covalent functionalization enables CNT's dispersion in different solvents, the process involves the chemical modification of SWCNTs. The conversion of, for example, $\mathrm{sp}^{2}$ carbons to $\mathrm{sp}^{3}$ carbons leads to the destruction of the extended $\pi$-system and, therefore, to changes in the resulting electronic properties. On the other hand, non-covalent interactions with molecular building blocks such as surfactants, which contain hydrophobic and hydrophilic domains, keep the carbon backbone of SWCNTs intact and preserve their electronic properties. The design of photoactive molecular building blocks capable of dispersing SWCNTs, while maintaining their delocalized $\pi$-electron systems intact, is of great importance, especially in the context of their use in solar energy conversion. ${ }^{13}$ 
(a)
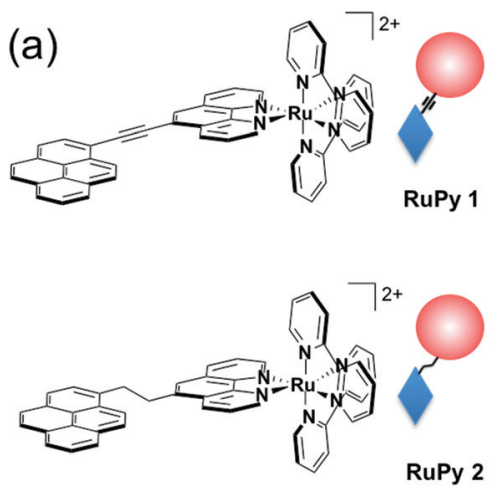

Scheme 1 (a) Structures of RuPy1 and RuPy2; (b) pictorial illustration of the interactions of RuPy1 and RuPy2 with SWCNTs.

Our group has previously reported on the use of ruthenium polypyridyl complexes with extended $\pi$-systems such as $[\mathrm{Ru}-$ $\left.(\text { bpy })_{2}(\mathrm{dppz})\right]^{2+}$ (bpy $=2,2^{\prime}$-bipyridine; dppz $=\operatorname{dipyrido}[3,2-$ $\left.a: 2^{\prime}, 3^{\prime}-c\right]$-phenazine) to non-covalently disperse SWCNTs in aqueous media. ${ }^{16}$ Although high concentration of SWCNTs are dispersible in aqueous solutions using $\left[\mathrm{Ru}(\mathrm{bpy})_{2}(\mathrm{dppz})\right]^{2+}$, its short-lived and non-emissive excited state in aqueous solution limits the excited state interactions between the metal complex and CNTs. ${ }^{21,22}$ In this study, we synthesized two ruthenium complexes containing pyrene-anchoring groups - Scheme 1. These complexes are photoluminescent in water with reasonably long photoluminescence lifetimes. ${ }^{23}$ Non-covalent $\pi$-stacking interactions between SWCNTs and pyrene are responsible for the physisorption of the photoluminescent complexes onto CNTs. The non-covalent attachment of pyrene derivatives onto SWCNTs not only keeps their electronic structure intact, but it allows the attachment of a variety of molecules to CNTs. ${ }^{2,24-28}$ In this manuscript, we demonstrate that ruthenium complexes containing pyrene-anchoring groups are efficient dispersers for SWCNTs in water. Using different spectroscopic techniques such as steady-state and time-resolved photoluminescence spectroscopy as well as transient absorption (pump-probe) spectroscopy, we probed the excited state of ruthenium complexes/SWCNTs composites in aqueous solution. The spectroscopic results obtained are consistent with quenching of the excited state by Förster Resonance Energy Transfer (FRET) from the ruthenium complexes to SWCNTs.

\section{Results and discussion}

Ruthenium complexes with pyrene anchoring groups (RuPy) were synthesized by multi-step reactions - see Schemes 2 and 3 - followed by ion exchange of the hexafluorophosphate salts with water-soluble chloride anions. Two molecules were synthesized labeled RuPy1 and RuPy2 as depicted in Scheme 1. The difference between RuPy1 and RuPy2 is the respective linker between the phenanthroline and the pyrene - for RuPy1 the linker is an alkyne group, while for RuPy 2 the linker is an alkane group.

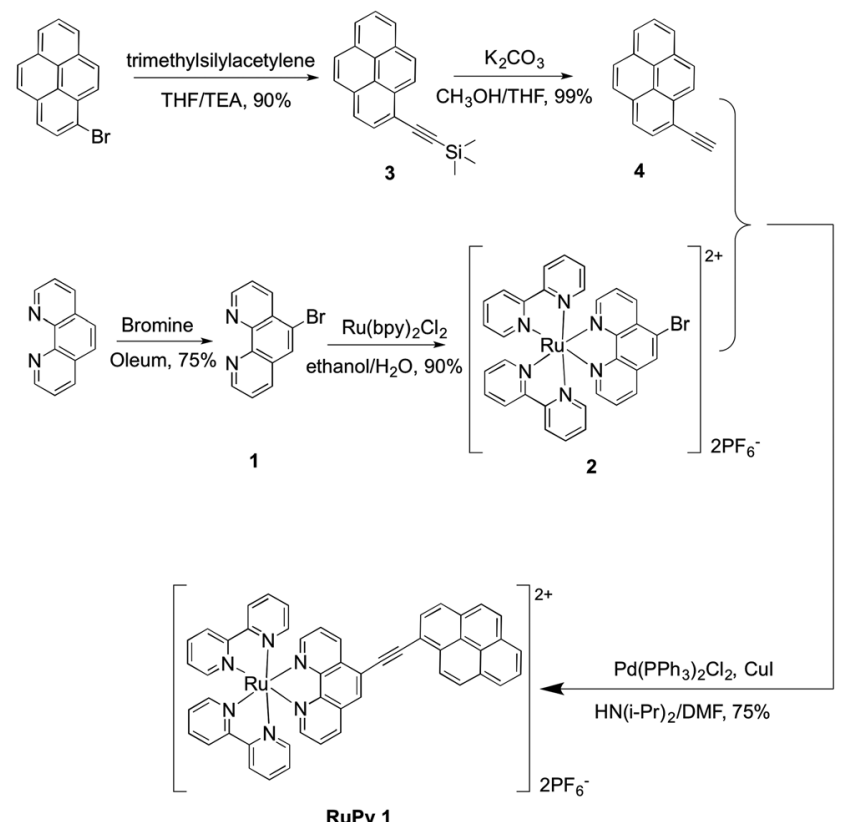

Scheme 2 Scheme for the synthesis of Rupy1.

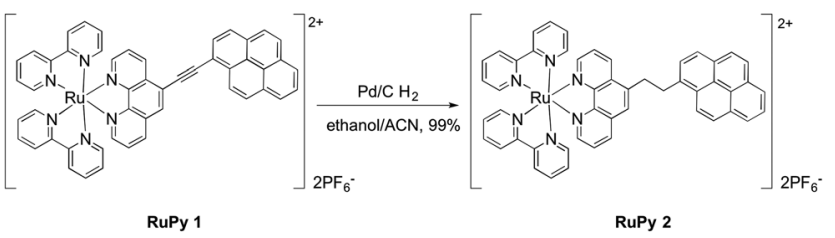

Scheme 3 Scheme for the synthesis of compound RuPy2.

The absorption and emission of RuPy1 and RuPy2 were studied in nitrogen purged aqueous solutions. The absorption spectrum of RuPy1 in Fig. 1 exhibits a broad absorption band with a maximum at $420 \mathrm{~nm}$, which possesses MLCT as well as IL character, and bands at 235 and $285 \mathrm{~nm}$, which correspond to ligand-centered transitions in line with a previous report of a similar complex. ${ }^{29}$ The existence of electronic communi-

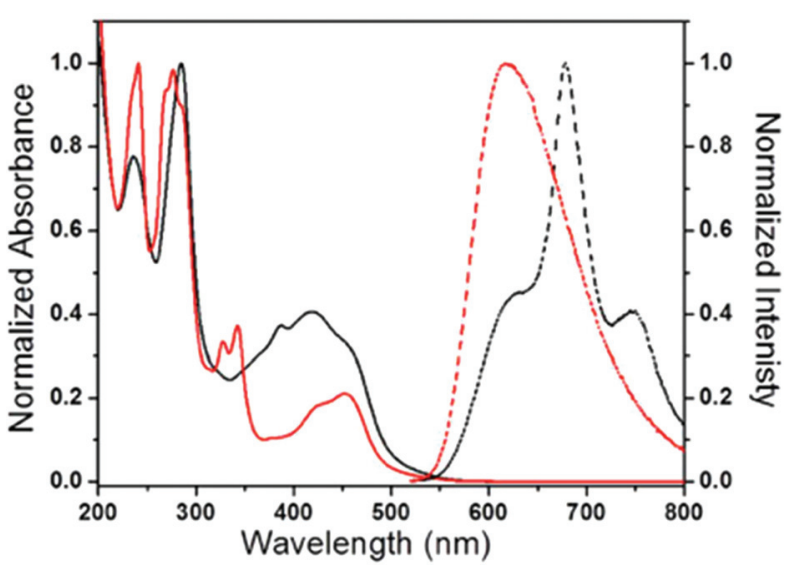

Fig. 1 Normalized absorption and emission spectra of RuPy1 (black spectra) and RuPy2 (red spectra) in nitrogen purged aqueous solution. 
cation between phenanthroline and pyrene via the triple bond conjugation in RuPy1 is the origin for a structured red-shifted emission with a maximum at $680 \mathrm{~nm}$. Interestingly, its emission lifetime in water reaches $665 \mu$ s. In accordance with previous studies, this phenomenon is attributed to the emission from ${ }^{3} \mathrm{IL} /{ }^{3} \mathrm{LLCT}$ excited states. ${ }^{29,30}$ RuPy2 shows an MLCT band with a maximum at $452 \mathrm{~nm}$ and distinguishable LC bands at higher energies. Reducing the acetylene group linking the phenanthroline and pyrene to afford RuPy2, disrupts the extended conjugation of the ligand and restores the typical shape of the MLCT seen in polypyridyl ruthenium complexes. ${ }^{31,32} \mathrm{~A}$ point to note is that RuPy2 has distinguishable peaks around $340 \mathrm{~nm}$, which resemble those of pyrene, and, which arise likely due to the disruption of conjugation upon reduction of the alkyne group. RuPy2 shows an unstructured emission at $615 \mathrm{~nm}$ consistent with the emission of $\left[\mathrm{Ru}(\mathrm{bpy})_{2}(\mathrm{phen})\right]^{2+}$ in water and an emission lifetime of $12 \mu$ s (Table S1 $\dagger$ ). The latter exceeds that of $\left[\mathrm{Ru}(\mathrm{bpy})_{2}(\mathrm{phen})\right]^{2+}$, which is consistent with a triplet-triplet excited state equilibrium between the ${ }^{3} \mathrm{MLCT}$ and pyrene centered ${ }^{3} \mathrm{IL}$ states. ${ }^{33-36}$

To study the interaction of RuPy complexes with a variety of nanotubes types (chiralities) we employed HiPco nanotubes. SWCNTs were dispersed in $\mathrm{D}_{2} \mathrm{O}$ solutions of either RuPy1 or RuPy2 with different initial amounts of HiPco SWCNTs. The resulting dispersions were tip sonicated followed by centrifugation to remove non-dispersed bundled SWCNTs. Then, the supernatant was collected to isolate dispersions of RuPy/HiPco SWCNT hybrids. Hybrids of RuPy with CoMoCAT SWCNTs were prepared in the same way. The absorption spectra of HiPco SWCNTs dispersed with RuPy1 are shown in Fig. 2,
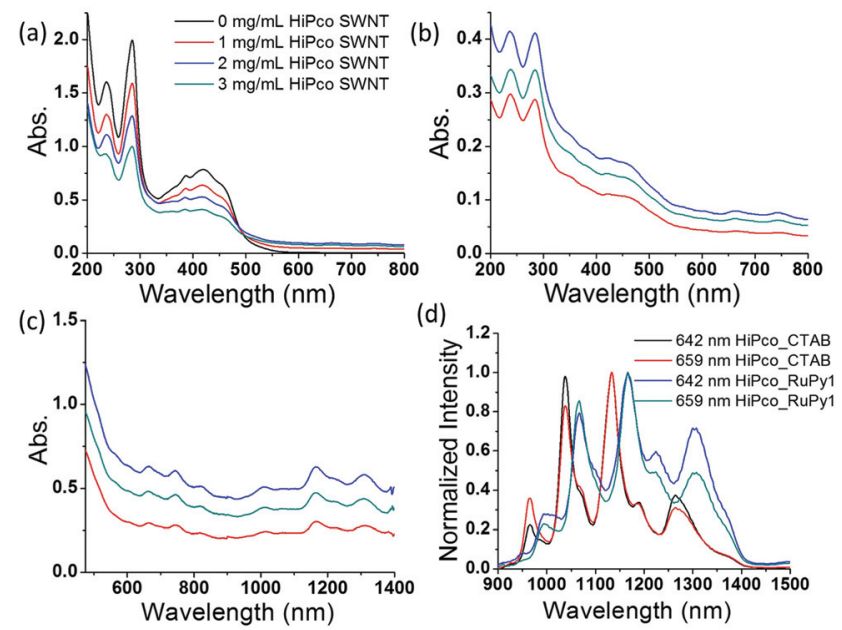

Fig. 2 Absorption spectra of RuPy1 and HiPco SWCNT hybrids in aqueous solutions before (a) and after (b) dialysis for $16 \mathrm{~h}$ using a nominal $2000 \mathrm{MW}$ membrane. Dispersions were prepared with $1 \mathrm{mg} \mathrm{mL}^{-1}$ RuPy1, with initial concentration of $0 \mathrm{mg} \mathrm{mL}^{-1}$ (black line), $1 \mathrm{mg} \mathrm{mL}^{-1}$ (red line), $2 \mathrm{mg} \mathrm{mL}^{-1}$ (blue line), and $3 \mathrm{mg} \mathrm{mL}^{-1}$ (cyan line) of HiPco SWCNTs, respectively (40x dilution before taking the spectra); (c) absorption spectra of RuPy1 and HiPco SWCNT hybrids in aqueous solution ( $5 \times$ dilution before taking the spectra); (d) comparison of photoluminescence spectra of HiPco SWCNTs dispersed in RuPy1 aqueous solution and CTAB surfactants. while those for RuPy2 are found in ESI, Fig. S2. $\dagger$ Consistent with Fig. 1, there is no appreciable absorption for RuPy1 above $550 \mathrm{~nm}$. On the contrary, for RuPy1/HiPco SWCNT hybrids, an increase in the absorption above 550 is noted (Fig. 2a), which shows the typical SWCNT centered van Hove transitions. We note in Fig. 2a that the absorbance at $420 \mathrm{~nm}$ decreases as the initial concentration of SWCNT increases, which implies the decrease of the RuPy1 concentration in solution. This phenomenon is attributed to the immobilization of RuPy1 onto non-dispersed SWCNTs following tip sonication and centrifugation. From analyses of Fig. 2a, we concluded that the optimum dispersion conditions for SWCNTs with RuPy1 include an initial concentration of $2 \mathrm{mg} \mathrm{mL}^{-1}$. Similar dispersion results are found for CoMoCAT SWCNTs, which are enriched in $(6,5)$ SWCNTs (Fig. S3†).

In order to study dispersed SWCNTs with minimum interference from free RuPy1 and/or RuPy2, the solutions were dialyzed using nominal $2000 \mathrm{MW}$ membranes. After dialysis, the absorption spectra of the resulting dispersions (Fig. 2b) give rise to a reduced amount of RuPy1 and/or RuPy2 as corroboration for the removal of free RuPy. In the near infrared, the absorption spectra of HiPco SWCNTs show discernable van Hove singularities, which is indicative of SWCNT individualization, and which underlines that $2 \mathrm{mg} \mathrm{mL}^{-1}$ is indeed the optimum initial concentration of SWCNTs when dispersed with RuPy1 (Fig. 2c). This confirms that dialysis for a controlled time period is a viable strategy for removing free RuPy1. If the dialysis continues beyond $24 \mathrm{~h}$, the dispersion is, however, rather unstable leading to the precipitation of SWCNTs. However, if the dialysis is stopped before or at the $24 \mathrm{~h}$ limit, the dispersions are stable for days. Notably, dispersions featuring lower concentrations of SWCNTs are dialyzable for more than $24 \mathrm{~h}$ without showing significant precipitation. Absorption spectra of RuPy2/HiPco SWCNTs are similar to those shown in Fig. 2 (Fig. S2b and S2c †). Qualitatively, the absorption spectra in Fig. $2 \mathrm{c}$ and $\mathrm{S} 2 \mathrm{c} \dagger$ seem to indicate that RuPy1 is slightly better dispersant for HiPco SWCNTs than RuPy2 in spite of its more rigid structure.

The individualization of SWCNTs in the dispersions is probed by the NIR emission of SWCNTs. Individualized semiconducting SWCNTs give rise to narrow emissions, whereas the emission is quenched in bundled SWCNTs possibly due to energy transfer to metallic nanotubes present in the bundles. ${ }^{37,38}$ Fig. 2d shows NIR emission of SWCNTs dispersed by RuPy1 and by cetyl hexadecyltrimethylammonium bromide (CTAB) and either excited at 642 or $659 \mathrm{~nm}$ in aqueous solutions. Again, the presence of emission peaks in the near infrared region attests their individualization. Substantial red-shifts in the order of $30 \mathrm{~nm}$ - in the emission maxima and line broadening were observed in RuPy1/HiPco SWCNTs when compared with CTAB/HiPco SWCNTs. This can be attributed to $\pi-\pi$ stacking of the pyrene anchors of RuPy1 onto the sidewalls of SWCNTs. ${ }^{16}$ Experiments using RuPy2 and SWCNTs show similar results (ESI, Fig. S2d †). Contour plots of the photoluminescence of dispersions of SWCNTs with RuPy1 and RuPy2 at different excitation wavelengths are shown in Fig. S5. $\dagger$ 


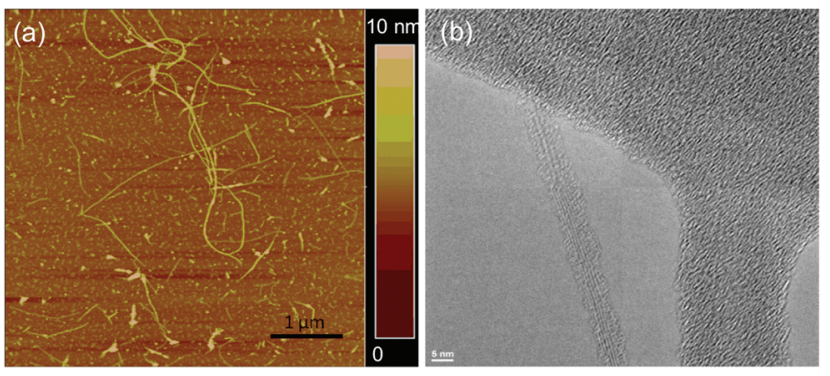

Fig. 3 (a) AFM image of spin coated RuPy1/HiPco SWCNT hybrids on mica and (b) TEM images of RuPy1/HiPco SWCNT hybrids on 200 mesh carbon type $\mathrm{B}$ coated copper grids.

Debundling of SWCNTs was independently confirmed by microscopy. Fig. 3a shows representative AFM images of RuPy1/HiPco SWCNTs deposited on mica. The average height of SWCNTs was estimated to be around $2.1 \mathrm{~nm}$, which corresponds to diameters of individual or mostly debundled HiPco SWCNTs covered with RuPy1. The morphology of RuPy1/HiPco SWCNTs was further visualized by TEM (Fig. 3b). Additional images are shown in ESI Fig. S6. $\dagger$ TEM images show two to three individual SWCNTs wrapped with an amorphous material, which suggests the immobilization of RuPy1 onto SWCNTs. Microscopy images of RuPy2/HiPco and RuPy1/CoMoCAT SWCNTs show similar features (Fig. S7-S9, $\dagger$ respectively).

In light of the fact that sonication induces defects on the sidewalls of SWCNTs, it is crucial to monitor the defects of dispersed SWCNTs. To this end, the G/D ratios in Raman spectra taken for SWCNTs are a qualitative approach to assess defects. Raman spectra of HiPco and CoMoCAT SWCNTs dispersed with RuPy1 were obtained by drop casting and drying the dispersions on silicon wafers (Fig. S10 $)$ ). In the spectra, G- and D-bands evolve at 1592 and $1300 \mathrm{~cm}^{-1}$, respectively. The relative intensity ratios of the G/D bands is 11.1, which infers slight damaging of the SWCNT sidewalls. In the literature, pristine SWCNTs feature G/D ratio in the range from 17 to $20 .^{8}$
To probe excited state interactions between SWCNTs and RuPy1 or RuPy2, we employed first steady-state emission spectroscopy. The effect of RuPy1 on the emission of HiPco SWCNTs was assessed by diluting dispersions of RuPy1/HiPco SWCNTs and CTAB/HiPco SWCNTs to the same absorption at $642 \mathrm{~nm}$. CTAB is a cationic surfactant, which should have minimum excited state interactions with SWCNTs. The NIR emission of SWCNTs excited at $642 \mathrm{~nm}$ was obtained and shows a 70\% quenching of the HiPco SWCNT emissions when dispersed with RuPy1, accompanied by a $30 \mathrm{~nm}$ red-shift in the emission maxima - Fig. 4a. The same experiments yield for RuPy2 a 94\% quenching of the HiPco SWCNTs centered features - Fig. S11a. $\dagger$ The quenching of the SWCNTs photoluminescence is consistent with quenching of the excited state by the heavy atom effect due to the close proximity of the ruthenium complexes. Actually, it is possible that the stronger quenching of SWCNT photoluminescence by RuPy2 is associated with a closer distance of this complex to the nanotube due to its flexible linker.

To study the effect of SWCNTs on the excited state emission of RuPy1, we prepared a RuPy1/HiPco SWCNTs dispersion with an absorbance of less than 0.05 at $440 \mathrm{~nm}$ to minimize inner filter effects. Then, we prepared a CTAB/HiPco SWCNTs dispersion and diluted it to match the absorption of RuPy1/ HiPco SWCNTs at $642 \mathrm{~nm}$ (this is done to estimate the contribution of SWCNT to the absorption spectrum of RuPy1/ HiPco). Implicit in the subtraction of these two spectra is the amount of RuPy1 used to disperse the HiPco SWCNTs. Then, we prepared a solution of RuPy1 with the same absorption at $420 \mathrm{~nm}$ as the spectra obtained by subtraction of the RuPy1 and RuPy1/SWCNTs spectra. This is carried out to guarantee the same concentrations of RuPy1 in both solutions. The emission of RuPy1 and RuPy1/SWCNTs were finally recorded showing a $92 \%$ decrease in intensity in the case of the latter Fig. 4b. The experiment for RuPy2 yields a 75\% emission quenching in the presence of HiPco SWCNTs - ESI, Fig. S11b. $\dagger$ The stronger quenching, as it is observed for RuPy1 with SWCNTs, is likely to be a consequence of its longer photo-
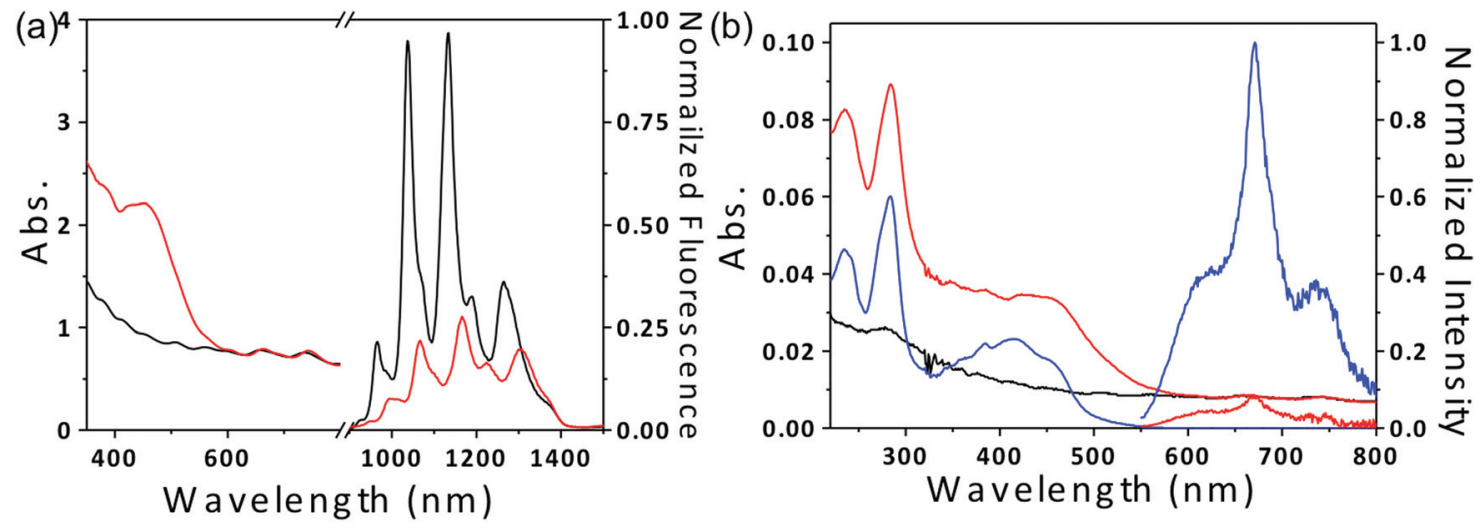

Fig. 4 (a) Absorption and NIR emission spectra of CTAB/HiPco SWCNT (black spectra) and RuPy1/HiPco SWCNT (red spectra) in aqueous solution upon $642 \mathrm{~nm}$ photoexcitation; (b) absorption and emission spectra of CTAB/HiPco SWCNT (black spectra), RuPy1/HiPco SWCNT (red spectra), and RuPy1 (blue spectra) in aqueous solutions upon $420 \mathrm{~nm}$ photoexcitation. 
luminescence lifetime in comparison with RuPy2 - vide infra. The nature of this quenching will be discussed in more detail below.

Emission lifetime analysis for RuPy1 also shows a decrease when SWCNTs are present, namely from 665 to $382 \mu \mathrm{s}$ (Table S2 $\dagger$ ). For RuPy2, the change in lifetime was from 12.2 to $6.8 \mu \mathrm{s}$. It is, however, important to mention that the quenching based on lifetime values is smaller $(\sim 55 \%)$ than what would be expected based on the steady state photoluminescence $(>75 \%$, see previous paragraph). As such, a static component must contribute to the quenching of the RuPy1 and RuPy2 emission in the presence of SWCNTs.

To further explore the nature of the SWCNTs and RuPy1 or RuPy2 interactions, pump-probe experiments were performed. Control pump-probe experiments of RuPy1 in $\mathrm{D}_{2} \mathrm{O}$ show bleaching at $450 \mathrm{~nm}$ due to the population of the ${ }^{3}$ MLCT, which recovers biexponentially with lifetimes of 8.5 and 102.5 ps - Fig. S12. $\dagger$ This bleach is followed by the emergence of a long-lived positive transient with maxima at 439 and $\sim 500 \mathrm{~nm}$, which are likely associated with triplet-triplet absorptions of pyrene. ${ }^{29}$ The differential absorption spectra for RuPy2 are similar to that of RuPy1. The only notable difference is the recovery of $450 \mathrm{~nm}$ bleaching, which exhibits lifetimes of 81.8 and 698.7 ps - Fig. S13. $\dagger$ This bleach, which is due to the population of the ${ }^{3}$ MLCT, is followed by the formation of positive transients with maxima at 490 and $515 \mathrm{~nm}$ (triplet-triplet absorptions of pyrene) as well as a negative transient at $\sim 630 \mathrm{~nm}$, which can be attributed to emission.

The pump-probe experiments for HiPco SWCNTs dispersed by CTAB were also obtained as control experiments and are shown in Fig. 5a. Two major features are observed in the pump-probe experiments: transient bleachings and transient absorption. Transient bleachings are due to pump laser excitation which creates $E_{i i}$ excitons that relax to $E_{11}$ within 100 fs. ${ }^{39}$ This produces a depletion of the ground state that is
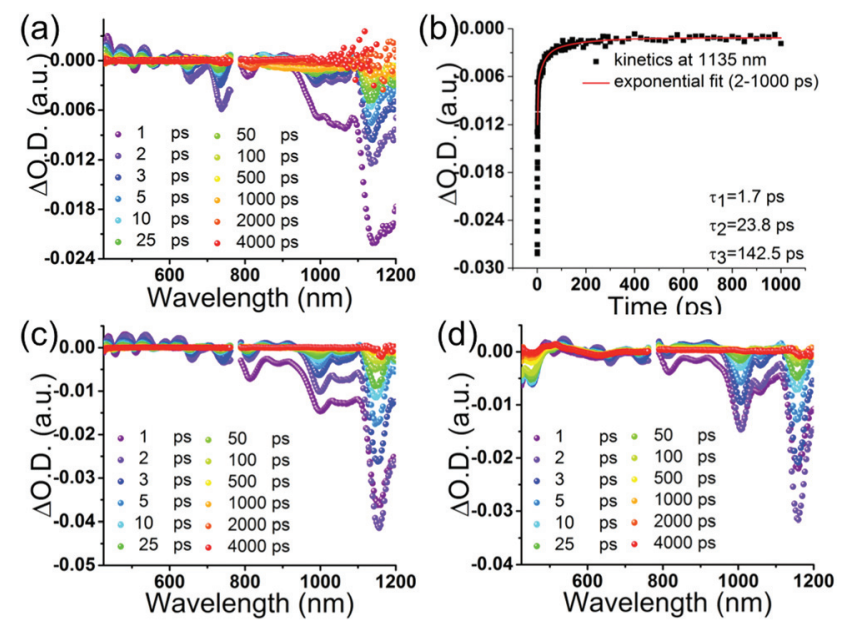

Fig. 5 Differential absorption spectra obtained upon femtosecond pump probe experiments $\left(387 \mathrm{~nm}\right.$ ) of (a) CTAB/HiPco SWCNTs in $\mathrm{D}_{2} \mathrm{O}$ with (b) its time decay profile at $1135 \mathrm{~nm}$. Differential absorption spectra for (c) RuPy1/HiPco SWCNTs and (d) RuPy2/HiPco SWCNTs in $\mathrm{D}_{2} \mathrm{O}$. observed as a bleaching in the van Hoff transitions. Transient absorption features have been attributed to several factors such as population of a biexciton manifold $\left(E_{11}+E_{22}\right)$ from $E_{11}$ and photoexcited bound excitons, ${ }^{39}$ e.g. $E_{22}-E_{11}$ or $E_{33}-$ $E_{11}{ }^{40}$ Transient bleachings are observed at 988, 1030, 1060, and $1140 \mathrm{~nm}$, which are consistent with bleaching of the ground state of $(6,5),(7,5),(10,2)$, and $(7,6)$ SWCNTs, respectively. These bleachings are red shifted in relation with HiPco nanotubes dispersed with SDBS. ${ }^{20}$ Multi exponential fits (see Fig. $5 \mathrm{~b}$ for fit of the $1135 \mathrm{~nm}$ transient) led to dynamics in the picosecond range of $\sim 2,24$, and 142 ps - Table S3. $\dagger$ Previous studies have suggested that short component lifetimes ( $\leq 2 \mathrm{ps})$ are due to relaxation of SWCNTs in bundles, ${ }^{40,41}$ although ultrafast relaxation could be also associated to exciton-exciton annihilation. ${ }^{42,43}$ Longer components from tens to hundreds of picoseconds can be assigned to band edge relaxation. ${ }^{41,44}$ It is important, however, to point out that exciton relaxation transition in SWCNTs depend on a variety of factors such as defects, length and dispersant. ${ }^{40}$

Pump-probe experiments for RuPy1/HiPco SWCNT show transient bleachings at 1000, $1050 \mathrm{~nm}$ (not prominent), and $1152 \mathrm{~nm}$ with similar lifetimes of $\sim 2,36$, and $169 \mathrm{ps}-$ Fig. 5c and Table S3. $\dagger$ For RuPy2/HiPco SWCNTs, the minima are further red shifted to 1005, 1055, $1156 \mathrm{~nm}$ - Fig. 5d - and with lifetimes as detailed in Table S3. $\uparrow$ Nonetheless, we did not observe any significant changes in the bi-exponential lifetimes in the excited state decay profile of RuPy1/HiPco SWCNTs and RuPy2/HiPco SWCNTs when compared to the analogous HiPco SWCNTs dispersion. Notable is that the transient absorption experiment for RuPy2/HiPco SWCNTs (Fig. 5d) show features that are consistent with free RuPy2 as (e.g. marked bleaching at $c a .450 \mathrm{~nm}$ ). This is because the samples for transient absorption experiments were not dialyzed in order to increase their stability during the transient absorption experiments (see Experimental section). These features are not obvious in the RuPy1/HiPco SWCNTs transient absorption experiments (Fig. 5c) due to the better interaction between RuPy1 and SWCNTs, which results in less free RuPy1.

In order to identify any potential new bands due to charge transfer interactions, we performed spectroelectrochemistry of RuPy1 and RuPy2. Fig. S14 and S15, ESI, $\dagger$ illustrate UV-Vis absorption spectra for the electrochemical oxidation and reduction of RuPy1 and RuPy2 upon applying variable potentials, respectively. On one hand, under oxidative and reductive conditions, attenuation of the MLCT related transitions is expressed in terms of negative differential absorption changes between 400 and $500 \mathrm{~nm}$. Once oxidized, the tendency to transfer metal $t_{2 g}$ electrons to ligand $\pi^{*}$ orbitals is eliminated. On the other hand, weak positive differential absorption changes in the range from 500 to $900 \mathrm{~nm}$ are in line with recent reports assigned to LMCT transitions. ${ }^{45}$ Unfortunately, the differential absorption bands seen at different reduction potentials resemble those of the control transient absorption experiments of the complexes in solution with no nanotubes (Fig. S12 and S13†). Comparison of Fig. 5c and d with Fig. 5a, indicate small shifts in maxima and intensities of the peaks, 

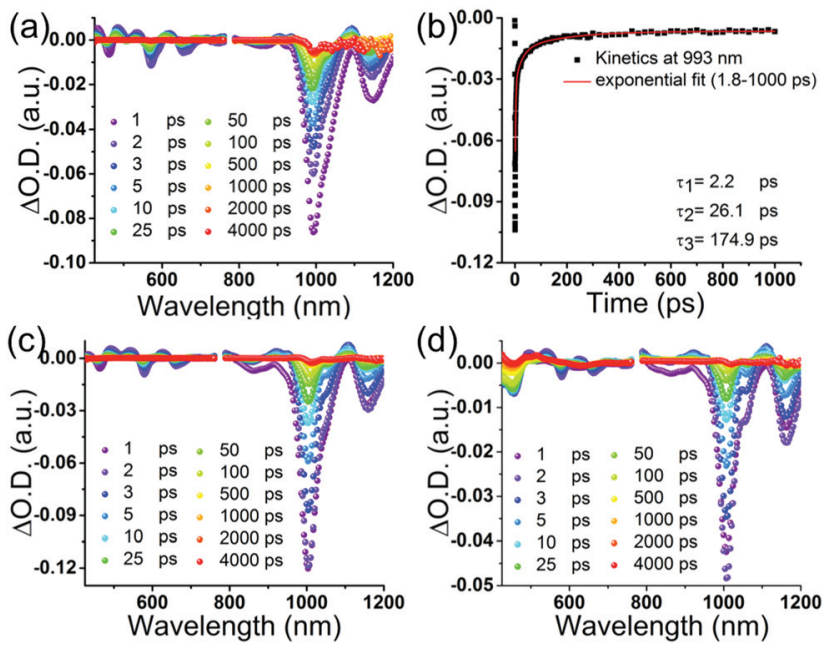

Fig. 6 Differential absorption spectra obtained upon femtosecond pump probe experiments $\left(387 \mathrm{~nm}\right.$ ) of (a) CTAB/COMoCAT in $\mathrm{D}_{2} \mathrm{O}$ with (b) its time-decay profile at $993 \mathrm{~nm}$. Differential absorption spectra for (c) RuPy1/CoMoCAT in $\mathrm{D}_{2} \mathrm{O}$, and (d) RuPy2/CoMoCAT in $\mathrm{D}_{2} \mathrm{O}$.

but no new spectral bands, which could be unambiguously associated with charge transfer processes (Fig. 5).

To overcome the heterogeneity of HiPco SWCNTs, we performed transient absorption experiments with CoMoCAT SWCNTs. The advantage of the CoMoCAT SWCNTs used is that they are enriched in $(6,5)$ nanotubes, allowing to obtain simplified signal pattern in the transient absorption experiment. Fig. 6a shows transient absorption spectra with minima at 993 and $1149 \mathrm{~nm}$ in the NIR range corresponding to $(6,5)$ and $(7,6)$ nanotubes dispersed via CTAB in $\mathrm{D}_{2} \mathrm{O}$. The ground state bleaching at, for example, $993 \mathrm{~nm}$ is fit to a triexponential decay with lifetimes of 2, 26, and 175 ps (Fig. 6b). Transient minima in the visible range at 464, 513, 571, $652 \mathrm{~nm}$, also corresponds to ground state bleaching, which match with the steady-state absorption spectrum. Transient maxima appear at 479, 530, 607, 902, 936, and $1093 \mathrm{~nm}$ and are in sound agreement with previous reports. ${ }^{20}$ The transient absorption spectra of CoMoCAT SWCNTs dispersed by means of RuPy1 are shown in Fig. 6c upon $387 \mathrm{~nm}$ excitation. Minima were observed in this case at 1003 and $1156 \mathrm{~nm}$, which correlate to ground state bleaching of $(6,5)$ and $(7,6)$ SWCNTs, respectively. Those bands are red-shifted in comparison with CoMoCAT SWCNTs. Exponential fitting of the dynamics resulted in three major lifetimes, namely 2, 19, and 136 ps for $(6,5)$ chirality. Interestingly, a biexponential decay was observed for $(7,6)$ tubes - Table S3. $\dagger$ In comparison, the minima in RuPy2/ CoMoCAT SWCNTs are slightly red shifted to $1006 \mathrm{~nm}$ and $1163 \mathrm{~nm}$ (Fig. 6d) with 2, 17, and 95 ps emerged as lifetimes for $(6,5)$. The red shifts observed for RuPy with SWCNTs are reminiscent of the red shifts observed in the photoluminescence spectra and are likely due to $\pi-\pi$ stacking.

Interestingly, in past works, both oxidation and reduction of SWCNTs in the presence of ruthenium polypyridyl complexes have been inferred based on photoconductivity, photo- voltaics performance, and FET studies. ${ }^{46-48}$ Notably, these studies have been performed in dry films, in which the orientation of ruthenium complexes relative to SWCNTs and/or the solvent reorganization energy is likely to play a role in the direction of electron density shifts. Thus, both directions of photo-excited electron transfer are in principle, thermodynamically feasible in RuPy/SWCNT hybrids as mentioned by Khairoutdinov et al. (oxidative and reductive quenching of $\mathrm{Ru}(\mathrm{bpy})_{3}{ }^{2+}$ by SWCNTs present favorable with $\Delta G^{\circ}$ of $-1.14 \mathrm{eV}$ and $-0.54 \mathrm{eV}$ respectively). ${ }^{46}$ Nonetheless, as discussed above for RuPy1 and RuPy2 with HiPco and CoMoCAT SWCNTs, no new features were observed in the transient absorption spectra, which would indicate photoredox chemistry. As such, the reason for the high degree of quenching cannot be attributed to photoinduced electron transfer.

A possibility so far unaddressed is that quenching of RuPy by SWCNTs could be due to FRET. Since the emission from RuPy1 and RuPy2 comes from an excited state with strong triplet character, FRET is spin-forbidden and, thus, it was expected to have minimum contribution to quenching. Nonetheless, calculations of the theoretical quenching efficiency between RuPy1 and $(6,5)$ SWCNTs as a function of the distance using FRETView (details in Appendix 1, ESI $\dagger$ ) shows that FRET is quite efficient when the distance between RuPy1 and SWCNT is short (Table S4 $\dagger$ ). From structural models we anticipate that the distance between the ruthenium cation and the center of a $(6,5)$ SWCNTs is about $1.25 \mathrm{~nm}$ (Fig. S16†), which correspond to an estimated FRET efficiency of unity.

Considering that the emission spectra of RuPy1 and RuPy2 are broad, overlapping with the absorption spectra of a variety of SWCNTs, and that the distance between these metal complexes and SWCNTs are expected to be very small, it is reasonable to think that quenching of the photoluminescence of RuPy1 and RuPy2 is due to FRET rather than electron transfer. Still, we must also point out that the expected FRET rate for RuPy1 to $(6,5)$ SWCNTs is slow $\left(10^{7}-10^{8} \mathrm{~s}^{-1}\right)$, which should yield an excited state lifetime component tens of nanoseconds long. Such a long-lived component was not detected in our transient absorption experiments, which could be taken as an argument against FRET. Nonetheless, it is likely that we are overestimating the distance between RuPy1 and $(6,5)$ SWCNTS in our calculation, which are really in van der Waals contact, and that would result in faster FRET rates (the RuPy1 excited state is localized on the pyrene group). Another possibility is a concerted electron exchange mechanism via Dexter energy transfer due to the close contact between the RuPy and SWCNTs. SWCNTs have a low-lying triplet state which could make a triplet-triplet Dexter mechanism possible. ${ }^{49}$ However, we did not observe the signature of long-lived triplet state due to Dexter energy transfer, at least in our experimental pumpprobe conditions. Other possibilities for the quenching of RuPy might include changes in the energy of pyrene excited state when bound to carbon nanotubes, which could lead to more efficient non-radiative deactivation pathways. It is also possible that quenching by charge transfer is still occurring, but with a charge recombination rate faster than the forward 
electron transfer, which would make its detection cumbersome by pump-probe spectroscopy. ${ }^{50}$

\section{Conclusions}

In conclusion, we have synthesized two ruthenium complexes - RuPy1 and RuPy2 - capable of dispersing SWCNTs in solution. The occurrence of SWCNT centred bandgap emission and microscopic assays suggest a great degree of individualization in the dispersions. Upon immobilization onto SWCNTs, the emission of both RuPy1 and RuPy2 decreases by up to $92 \%$. Transient absorption experiments show features consistent with RuPy1 and RuPy2 as well as SWCNT excitation, but no new bands corresponding to photoinduced redox species were unambiguously identified. Calculation of FRET parameters indicated that energy transfer can occur in this system with an efficiency of unity. This calculated FRET efficiency, combined with the lack of evidence for photoredox species from the pump-probe experiments lead to the conclusion that the quenching of RuPy1 and RuPy2 in the presence of SWCNTs must be due to FRET. Due to the large body of research on ruthenium polypyridine complexes electron donor-acceptor systems for solar energy conversion and water splitting, ${ }^{31,32,51,52}$ the creation of water-soluble RuPy/SWCNTs present interesting systems with potential use in artificial photosynthetic schemes. Since the proximity of the pyrene group to the $\mathrm{Ru}(\mathrm{II})$ complex produces a very short lived MLCT, future work will include the creation of ruthenium complexes containing long aliphatic chains instead of pyrene groups, which will allow a better monitoring of the long-lived MLCT band in the transient absorption spectrum and a more concise study of their photoredox chemistry.

\section{Experimental section}

${ }^{1} \mathrm{H}$ NMR spectra were recorded with a Bruker $400 \mathrm{MHz}$ NMR spectrometer. Electrospray ionization mass spectra (ESI-MS) were measured on a Bruker MicroTOF system. UV-Vis spectra absorption were recorded on a Shimadzu UV-2450 UV-Vis spectrophotometer. Steady-state photoluminescence spectra (in the visible region of the spectrum) were obtained at a HORIBA JovinYvonFluorolog 3 . Fluorescence lifetime measurements were performed using an Edinburgh Instruments OD470 single-photon counting spectrometer equipped with a blue detector and $\mu \mathrm{F} 920 \mathrm{H}$ lamp with excitation at $420 \mathrm{~nm}$ for RuPy1, while a $440 \mathrm{~nm}$ picosecond pulse diode laser and a high speed red detector was used for RuPy2. 2D steady-state photoluminescence spectra (in the NIR detection) were measured with a model NS1 Nanospectralyzer (Applied NanoFluorescence) with fixed excitation lasers at 642, and $659 \mathrm{~nm}$. Cyclic voltammetry and square wave voltammetry were performed using a FRA $2 \mu$ Autplab type III (METROHM) potentiostat, in a conventional three-electrode electrochemical cell. A glassy carbon disk ( $1 \mathrm{~mm}$ diameter) was used as working elec- trode, a Pt wire electrode served as the counter electrode, an $\mathrm{Ag}$ wire was used as the quasi reference electrode while $(n-\mathrm{Bu})_{4} \mathrm{NPF}_{6}$ was used as the supporting electrolyte. All potentials were referenced to ferrocenium/ferrocene $\left(\mathrm{Fc}^{+} / \mathrm{Fc}\right)$. All electrochemical measurements were performed after purging with argon at ambient temperature. Spectroelectrochemical experiments were performed also with a three-electrode setup in a home-built cell - equipped with a platinum gauze as working electrode, a Pt wire as the counter electrode, and a Ag wire as quasi reference electrode. Variable potentials were applied using a PGSTAT 101 and the results were shown as differential spectra - spectrum with and without applied potential. The spectra were monitored with a Cary 5000 (VARIAN) UV/vis/NIR spectrometer. In case of RuPy/CoMoCAT, $\mathrm{NaCl}$ is used as supporting electrolyte whereas conventional $(n-\mathrm{Bu})_{4} \mathrm{NPF}_{6}$ is used for spectroelectrochemistry measurements of RuPy1 and 2 complexes. Raman spectra were obtained using a Renishaw inVia MicroRaman spectrometer. AFM images were taken on a scanning probe microscope, Digital instrument nanoscope III. TEM images were obtained from JEOL 2100 FEG TEM (200 kV, high resolution) and LEO 912 OMEGA transmission electron microscope $(80 \mathrm{kV})$. Femtosecond transient absorption studies were performed with $387 \mathrm{~nm}$ laser pump pulses $(1 \mathrm{kHz}, 150 \mathrm{fs}$ pulse width - output: $775 \mathrm{~nm}$ ) from an amplified Ti-sapphire laser system (CPA 2110 Laser, Clark-MXR Inc.). Transient absorption spectra were monitored in $2 \mathrm{~mm}$ cuvettes with a Helios TAPPS setup. 3D steadystate photoluminescence spectra (in the NIR detection) were taken with a FluroLog3 spectrometer (Horiba) with InGaAs detectors (IGA Symphony 800 to $1700 \mathrm{~nm} 512 \times 1 \times 1 \mu \mathrm{m}$ ).

\section{Synthetic procedures}

All reagents and chemicals were obtained from commercial sources and used as received without purification. cis-Dichlorobis(2,2'-bipyridine)ruthenium(II) dehydrate was purchased from Strem Chemicals. 1-Bromopyrene, 1,10-phenanthroline, and (trimethylsilyl)acetylene were purchased from Alfa Aesar, USA. Potassium hexafluorophosphate was purchased from TCI America. All other chemicals were purchased from SigmaAldrich. Dialysis membranes with Nominal MWCO 2000 were purchased from Membrane Filtration Products, Inc.

Synthesis of compound 1. This compound was synthesized based on literature methods..$^{53} 2.4 \mathrm{~g}$ of 1,10-phenanthroline were placed in a heavy wall glass reaction tube with a Teflon screw top fitted with a Viton-O-ring. The reaction vessel was placed in an ice bath, and $8 \mathrm{~mL}$ of oleum (18\%) and $0.4 \mathrm{~mL}$ of bromine were added. The reaction tube was placed in a silicon oil bath, and the temperature was slowly raised to $135^{\circ} \mathrm{C}$. After $24 \mathrm{~h}$, the reaction mixture was cooled to room temperature, poured over ice, and neutralized with ammonium hydroxide. The mixture was extracted with chloroform. The red extracts were stirred with activated charcoal and then dried over sodium sulfate. The crude product was recrystallized from hot diethyl ether with a little amount of dichloromethane. ${ }^{1} \mathrm{H}$ NMR $\left(400 \mathrm{MHz}, \mathrm{CDCl}_{3}\right) \delta(\mathrm{ppm}) 9.214(\mathrm{t}, J=4.8,2 \mathrm{H}), 8.675$ 
$(\mathrm{d}, J=8.4,1 \mathrm{H}), 8.184(\mathrm{~d}, J=8.0,1 \mathrm{H}), 8.150(\mathrm{~s}, 1 \mathrm{H}), 7.750(\mathrm{dd}$, $J=4.4,1 \mathrm{H}), 7.652(\mathrm{dd}, J=4.4,1 \mathrm{H})$.

Synthesis of compound 2. This compound was synthesized based on literature methods. ${ }^{54}$ The mixture of cis-dichlorobis (2,2'-bipyridine)ruthenium(II) dehydrate $(0.260 \mathrm{~g})$ and compound $1(0.130 \mathrm{mg})$ in ethanol and water mixture solvent $(\mathrm{v} / \mathrm{v}$, 1/1) was heated to reflux for 2 hours. After this, it was cooled down to room temperature and $150 \mathrm{mg}$ of potassium hexafluorophosphate were added. The solvent was removed by rotovap and the crude product was purified by silica gel (ACN : $\mathrm{CH}_{2} \mathrm{Cl}_{2}$ 1:4) in 75\% yield. ${ }^{1} \mathrm{H} \mathrm{NMR}\left(400 \mathrm{MHz}, \mathrm{CD}_{3} \mathrm{CN}\right)$ $\delta(\mathrm{ppm}) 8.742(\mathrm{~d}, J=8.4,1 \mathrm{H}), 8.742(\mathrm{~d}, J=8.4,1 \mathrm{H})$, 8.602-8.538 (overlapping $\mathrm{m}, 6 \mathrm{H}$ ), 8.202 (dd, $J=6.8,2 \mathrm{H}$ ), 8.116 (t, $J=8.0,2 \mathrm{H}), 8.024(\mathrm{t}, J=8.4,2 \mathrm{H}), 7.903(\mathrm{~m}, 2 \mathrm{H}), 7.850(\mathrm{dd}$, $J=5.2,1 \mathrm{H}), 7.781(\mathrm{dd}, J=5.2,1 \mathrm{H}), 7.610(\mathrm{~m}, 2 \mathrm{H}), 7.489(\mathrm{t}, J=$ 6.4, 2H), $7.280(\mathrm{~m}, 2 \mathrm{H})$. ESI-MS: $m / z 817.0\left(\mathrm{M}-\mathrm{PF}_{6}{ }^{-}\right), 337.0$ $\left(\left[\mathrm{M}-2 \mathrm{PF}_{6}^{-}\right] / 2\right)$.

Synthesis of compound 3 . This compound was synthesized based on literature methods. ${ }^{55}$ A mixture of 1-bromopyrene (560 mg), $\mathrm{Pd}\left(\mathrm{PPh}_{3}\right)_{2} \mathrm{Cl}_{2}(175 \mathrm{mg})$, CuI (50 mg) and piperidine $(4 \mathrm{~mL})$ in $30 \mathrm{~mL}$ triethylamine was bubbled with nitrogen for 20 minutes. Then, the trimethylsilylacetylene was added and the resulting solution was heated at $110{ }^{\circ} \mathrm{C}$ overnight under nitrogen protection. The mixture was evaporated by rotovap and the residue was subjected to column chromatography on silica gel, eluting with hexane to yield a yellow crystalline product. ${ }^{1} \mathrm{H}$ NMR (400 $\left.\mathrm{MHz}, \mathrm{CDCl}_{3}\right) \delta$ (ppm) 8.567 (d, $J=9.2$, 1H), 8.228-8.002 (overlapping $\mathrm{m}, 8 \mathrm{H}$ ), 0.395 (s, 9H).

Synthesis of compound 4 . This compound was synthesized based on literature methods. ${ }^{55}$ The mixture solution of compound 3 (300 $\mathrm{mg}$ ) and potassium bicarbonate $(690 \mathrm{mg}$ ) was stirred at room temperature for $1 \mathrm{~h}$, the solvent removed, and the crude compound further purified by column chromatography on silica gel, eluting with hexane and dichloromethane mixture $(\mathrm{v} / \mathrm{v}, 10 / 1)$. Since compound $\mathbf{4}$ is oxygen and light sensitive, it was used directly for next step without any storage.

Synthesis of compound RuPy1. This compound was synthesized based on literature methods. ${ }^{29}$ Compound 2 (100 mg), compound 4 (46 mg), $\mathrm{Pd}\left(\mathrm{PPh}_{3}\right)_{2} \mathrm{Cl}_{2}$ (35 mg) and $\mathrm{CuI}$ $(9.8 \mathrm{mg})$ were added to a $50 \mathrm{~mL}$ round bottom flask. To this flask $2 \mathrm{~h}$ purged DMF and $\mathrm{HN}(\mathrm{i}-\mathrm{Pr})_{2}$ mixture solvent $(10 \mathrm{~mL}$, $\mathrm{v} / \mathrm{v}, 3 / 2)$ were added. The solution was stirred under nitrogen protection at room temperature for $24 \mathrm{~h}$. After that, the mixture was filtered and the liquid filtrate was concentrated under reduced pressure. The crude compound was further purified by column chromatography on silica gel with acetonitrile and dichloromethane mixture $(\mathrm{v} / \mathrm{v}, 1 / 4)$ as eluent. ${ }^{1} \mathrm{H}$ NMR $(400 \mathrm{MHz}, \mathrm{CD} 3 \mathrm{CN}) \delta(\mathrm{ppm}) 9.130(\mathrm{~d}, J=8.0,1 \mathrm{H}), 8.750(\mathrm{~d}, J=$ 9.2, 1H), 8.628-8.527 (m, 6H), $8.393(\mathrm{~m}, 3 \mathrm{H}), 8.297$ (d, $J=8.0$, $1 \mathrm{H}), 8.267$ (d, $J=7.6,1 \mathrm{H}), 8.209(\mathrm{~d}, J=5.2,1 \mathrm{H}), 8.167-8.020$ (overlapping $\mathrm{m}, 8 \mathrm{H}), 7.906(\mathrm{~m}, 3 \mathrm{H}), 7.793(\mathrm{dd}, J=8.4 ; 5.6,1 \mathrm{H})$, $7.680(\mathrm{~d}, J=5.6,1 \mathrm{H}), 7.621(\mathrm{~d}, J=6.0,1 \mathrm{H}), 7.499(\mathrm{t}, J=6.4$, $2 \mathrm{H}$ ), 7.303 (quint, 2H). ESI-MS: $m / z 963.1\left(\mathrm{M}-\mathrm{PF}_{6}{ }^{-}\right), 409.1$ $\left(\left[\mathrm{M}-2 \mathrm{PF}_{6}{ }^{-}\right] / 2\right)$. High-Resolution MS: $m / z 963.1379\left(\mathrm{M}-\mathrm{PF}_{6}{ }^{-}\right)$, $409.0868\left(\left[\mathrm{M}-2 \mathrm{PF}_{6}^{-}\right] / 2\right)$.
Synthesis of compound RuPy2. RuPy1 (100 mg) and Pd/C $(20 \mathrm{mg})$ as catalyst were added to a $100 \mathrm{~mL}$ three-necked round bottom flask under a hydrogen atmosphere. Then, a $2 \mathrm{~h}$ Argon purged ethanol and acetonitrile mixture solvent (v/v, 1/4) were added. The solution was stirred at room temperature for $24 \mathrm{~h}$ under hydrogen. After that, the precipitate was removed by filtration (twice) and the liquid filtrate was collected under reduced pressure. The same reduction process was repeated twice to make sure all the RuPy1 was reduced into RuPy2. The product was used directly for ionic exchange. ${ }^{1} \mathrm{H}$ NMR $\left(400 \mathrm{MHz}, \mathrm{CD}_{3} \mathrm{CN}\right) \delta(\mathrm{ppm}) 8.664(\mathrm{~d}, J=8.8,1 \mathrm{H}), 8.531(\mathrm{~m}$, $4 \mathrm{H}), 8.392(\mathrm{~d}, J=8.0,1 \mathrm{H}), 8.328(\mathrm{~d}, J=9.2,1 \mathrm{H}), 8.238(\mathrm{t}, J=$ 6.8, 2H), 8.132-8.017 (overlapping $\mathrm{m}, 12 \mathrm{H}$ ), $7.856(\mathrm{~d}, J=5.6$, $2 \mathrm{H}), 7.825(\mathrm{~d}, J=8.0,1 \mathrm{H}), 7.661(\mathrm{q}, 1 \mathrm{H}), 7.578(\mathrm{q}, 1 \mathrm{H}), 7.521(\mathrm{t}$, $J=6.4,2 \mathrm{H}), 7.461(\mathrm{t}, J=7.2,2 \mathrm{H}), 7.255(\mathrm{~m}, 2 \mathrm{H}), 3.909(\mathrm{t}, 2 \mathrm{H})$, $3.822(\mathrm{t}, 2 \mathrm{H})$. ESI-MS: $m / z 967.1\left(\mathrm{M}-\mathrm{PF}_{6}{ }^{-}\right), 411.1([\mathrm{M}-$ $\left.\left.2 \mathrm{PF}_{6}^{-}\right] / 2\right)$.

Ion exchange of the RuPy1 and 2 hexafluorophosphate salts to chloride salts. The $\mathrm{PF}_{6}$ salts were dissolved in acetone as concentrated as possible, followed by dropwise addition of a saturated solution of tetra- $n$-butylammonium chloride (TBACl) in acetone. The orange-red chloride salts was precipitated, collected by centrifugation in several $1.5 \mathrm{~mL}$ centrifuge tubes, and washed with large amounts of ethyl acetate to wash away extra TBACl.

Dispersion of SWCNTs. Raw SWCNTs were purified as reported elsewhere. ${ }^{56}$ Different amount of purified HiPco (Batch \# 195.1) and CoMoCAT (Sigma-Aldrich) SWCNTs were weighted in separate vials, followed by addition of $1 \mathrm{mg} \mathrm{ml} \mathrm{m}^{-1}$ RuPy1 aqueous solution to give different initial concentration of the nanotube in solution. The resultant mixture was sonicated with a probe sonicator (Sartorius, LABSONIC® M) for 30 minutes and then centrifuged (VWR centrifugator) for 45 minutes at $12000 \mathrm{~g}$. The supernatant was collected carefully and diluted for further analysis.

For transient absorption measurements, $\sim 0.2-0.3 \mathrm{mg}$ of HiPco and CoMoCAT tubes were added to RuPy1 $\left(2.5 \times 10^{-4}\right.$ M) and RuPy2 $\left(5 \times 10^{-4} \mathrm{M}\right)$ solutions in $\mathrm{D}_{2} \mathrm{O}$ respectively. The resulting mixtures were kept in a temperature-controlled ultrasonic bath for $20 \mathrm{~min}$ at $37 \mathrm{kHz}$ (Elmasonic P120 H, pulse mode). The dispersions were centrifuged at $12000 \mathrm{~g}$ for 45 minutes to remove large bundles. The sonication/centrifugation steps were repeated once again with the addition of nanotubes to enrich the amount of dispersed SWCNTs as described previously. ${ }^{20}$ Finally, the dispersion of centrifuged nanotubes was used for transient absorption measurements. Transmission electron microscopy measurement showed debundled SWCNTs of comparable diameter ( $c a .2 .1-2.4 \mathrm{~nm}$ ) by both tip sonication and bath sonication procedures.

Dialysis. For UV-Vis and fluorescence experiments, the free ruthenium complexes were removed by dialysis. The dialysis procedure was performed using Nominal MWCO 2000 membranes (Cellu Sep H1 and part \# 5-0250-28). $3 \mathrm{~mL}$ of the dispersed nanotubes solution were added to the dialysis membrane. Either side of the membrane was sealed by a dialysis tubing clip. The membrane bag was stirred gently in HPLC grade water, 
which was changed every 8 hour. After 24 hours, the RuPy1/ SWCNT composites solution was removed from the dialysis membrane and centrifuged again for 45 minutes at $12000 \mathrm{~g}$. The supernatant was collected and used in the experiments.

AFM and TEM sample preparation. For AFM (Digital instrument nanoscope) a RuPy1/SWCNT dispersions (HiPco and CoMoCAT) were diluted 10 times and $10 \mu \mathrm{L}$ of the solution was drop casted on freshly cleaved mica and spin coated at $4000 \mathrm{rpm}$ for 15 minutes. During spinning, the sample was washed with 4-5 drops of methanol to remove excess $\mathrm{Ru}$ complexes. To prepare the TEM sample, diluted RuPy1/SWCNT solutions were drop casted on lacey carbon grids and dried overnight. Jeol 2100 field emission gun (FEG) TEM was used to visualize the nanotubes.

\section{Acknowledgements}

The Welch foundation grant C-1743 (A.A.M.) and SFB 953 (D.F.G.) are acknowledged for financial support. We acknowledge Leonie Wibmer (Friedrich-Alexander-Universität Erlangen-Nürnberg) for recording Raman spectra.

\section{Notes and references}

1 S. Iijima, Nature, 1991, 354, 56-58.

2 J. Bartelmess, B. Ballesteros, G. de la Torre, D. Kiessling, S. Campidelli, M. Prato, T. Torres and D. M. Guldi, J. Am. Chem. Soc., 2010, 132, 16202-16211.

3 Y. Jung, X. Li, N. K. Rajan, A. D. Taylor and M. A. Reed, Nano Lett., 2013, 13, 95-99.

4 D. D. Tune and J. G. Shapter, Energy Environ. Sci., 2013, 6, 2572-2577.

5 R. H. Lau, K. Takei, C. Wang, Y. Ju, J. Kim, Z. Yu, T. Takahashi, G. Cho and A. Javey, Nano Lett., 2013, 13, 3864-3869.

6 F. Xu, M.-Y. Wu, N. S. Safron, S. S. Roy, R. M. Jacobberger, D. J. Bindi, J.-H. Seo, T.-H. Chang, Z. Ma and M. S. Arnold, Nano Lett., 2014, 14, 682-686.

7 A. G. Nasibulin, A. Kaskela, F. Mustonen, A. S. Anisimov, V. Ruiz, S. Kivistö, S. Rackauskas, M. Y. Timmermans, M. Pudas, B. Aitchison, M. Kauppinen, D. P. Brown, O. G. Okhotnikov and E. I. Kauppinen, ACS Nano, 2011, 5, 3214-3221.

8 A. Saha, S. Ghosh, R. B. Weisman and A. A. Martí, ACS Nano, 2012, 6, 5727-5734.

9 B. Rösner, D. M. Guldi, J. Chen, A. I. Minett and R. H. Fink, Nanoscale, 2014, 6, 3695-3703.

10 N. Behabtu, C. C. Young, D. E. Tsentalovich, O. Kleinerman, X. Wang, A. W. K. Ma, A. Bengio, R. F. ter Waarbeek, J. J. de Jong, R. E. Hoogerwerf, S. B. Fairchild, J. B. Ferguson, B. Maruyama, J. Kono, Y. Talmon, Y. Cohen, M. J. Otto and M. Pasquali, Science, 2013, 339, 182-186.
11 C. Jiang, A. Saha, C. C. Young, D. P. Hashim, C. E. Ramirez, P. M. Ajayan, M. Pasquali and A. A. Martí, ACS Nano, 2014, 8, 9107-9112.

12 A. D. Leonard, J. L. Hudson, H. Fan, R. Booker, L. J. Simpson, K. J. O’Neill, P. A. Parilla, M. J. Heben, M. Pasquali, C. Kittrell and J. M. Tour, J. Am. Chem. Soc., 2009, 131, 723-728.

13 D. M. Guldi, G. M. A. Rahman, N. Jux, N. Tagmatarchis and M. Prato, Angew. Chem., Int. Ed., 2004, 43, 5526-5530.

14 C. Jiang, A. Saha, X. Changsheng, C. Young, J. M. Tour, M. Pasquali and A. A. Martí, ACS Nano, 2013, 7, 4503-4510.

15 A. Saha, S. Ghosh, N. Behabtu, M. Pasquali and A. A. Martí, Chem. Sci., 2011, 2, 1682-1687.

16 D. Jain, A. Saha and A. A. Martí, Chem. Commun., 2011, 47, 2246-2248.

17 Z. Chen, K. Kobashi, U. Rauwald, R. Booker, H. Fan, W.-F. Hwang and J. M. Tour, J. Am. Chem. Soc., 2006, 128, 10568-10571.

18 F. Hof, S. Bosch, S. Eigler, F. Hauke and A. Hirsch, J. Am. Chem. Soc., 2013, 135, 18385-18395.

19 Y.-L. Zhao and J. F. Stoddart, Acc. Chem. Res., 2009, 42, 1161-1171.

20 C. Romero-Nieto, R. García, M. A. Herranz, C. Ehli, M. Ruppert, A. Hirsch, D. M. Guldi and N. Martín, J. Am. Chem. Soc., 2012, 134, 9183-9192.

21 E. J. C. Olson, D. Hu, A. Hörmann, A. M. Jonkman, M. R. Arkin, E. D. A. Stemp, J. K. Barton and P. F. Barbara, J. Am. Chem. Soc., 1997, 119, 11458-11467.

22 J. Olofsson, B. Önfelt, P. Lincoln, B. Nordén, P. Matousek, A. W. Parker and E. Tuite, J. Inorg. Biochem., 2002, 91, 286-297.

23 S. Monro, J. Scott, A. Chouai, R. Lincoln, R. Zong, R. P. Thummel and S. A. McFarland, Inorg. Chem., 2010, 49, 2889-2900.

24 F. J. Gómez, R. J. Chen, D. Wang, R. M. Waymouth and H. Dai, Chem. Commun., 2003, 190-191.

25 C. Ehli, G. M. A. Rahman, N. Jux, D. Balbinot, D. M. Guldi, F. Paolucci, M. Marcaccio, D. Paolucci, M. Melle-Franco, F. Zerbetto, S. Campidelli and M. Prato, J. Am. Chem. Soc., 2006, 128, 11222-11231.

26 Y.-L. Zhao, L. Hu, J. F. Stoddart and G. Grüner, Adv. Mater., 2008, 20, 1910-1915.

27 F. D'Souza, A. S. D. Sandanayaka and O. Ito, J. Phys. Chem. Lett., 2010, 1, 2586-2593.

28 M. Yu, S.-Z. Zu, Y. Chen, Y.-P. Liu, B.-H. Han and Y. Liu, Chem. - Eur. J., 2009, 16, 1168-1174.

29 S. Ji, W. Wu, W. Wu, P. Song, K. Han, Z. Wang, S. Liu, H. Guo and J. Zhao, J. Mater. Chem., 2010, 20, 1953-1963.

30 S. Ji, W. Wu, W. Wu, H. Guo and J. Zhao, Angew. Chem., Int. Ed., 2011, 50, 1626-1629.

31 K. Kalyanasundaram, Coord. Chem. Rev., 1982, 46, 159-244.

32 K. Kalyanasundaram, Photochemistry of Polypyridine and Phorphyrin Complexes, Academic Press, London, 1992.

33 N. D. McClenaghan, Y. Leydet, B. Maubert, M. T. Indelli and S. Campagna, Coord. Chem. Rev., 2005, 249, 1336-1350.

34 M. Hissler, A. Harriman, A. Khatyr and R. Ziessel, Chem. Eur. J., 1999, 5, 3366-3381. 
35 D. S. Tyson, K. B. Henbest, J. Bialecki and F. N. Castellano, J. Phys. Chem. A, 2001, 105, 8154-8161.

36 G. J. Wilson, A. Launikonis, W. H. F. Sasse and A. W.-H. Mau, J. Phys. Chem. A, 1997, 101, 4860-4866.

37 J. Lefebvre and P. Finnie, J. Phys. Chem. C, 2009, 113, 75367540.

38 O. N. Torrens, D. E. Milkie, M. Zheng and J. M. Kikkawa, Nano Lett., 2006, 6, 2864-2867.

39 B. Gao, G. V. Hartland and L. Huang, ACS Nano, 2012, 6, 5083-5090.

40 J.-P. Yang, M. M. Kappes, H. Hippler and A.-N. Unterreiner, Phys. Chem. Chem. Phys., 2005, 7, 512-517.

41 I. V. Rubtsov, R. M. Russo, T. Albers, P. Deria, D. E. Luzzi and M. J. Therien, Appl. Phys. A, 2004, 79, 1747-1751.

42 Y.-Z. MA, L. Valkunas, S. L. Dexheimer, S. M. Bachilo and G. R. Fleming, Phys. Rev. Lett., 2005, 94, 157402.

43 L. Lüer, S. Hoseinkhani, D. Polli, J. Crochet, T. Hertel and G. Lanzani, Nat. Phys., 2009, 5, 54-58.

44 L. Huang, H. N. Pedrosa and T. D. Krauss, Phys. Rev. Lett., 2004, 93, 017403-017401.

45 A. M. Brown, C. E. McCusker and J. K. McCusker, J. Chem. Soc., Dalton Trans., 2014, 43, 17635-17646.
46 R. F. Khairoutdinov, L. V. Doubova, R. C. Haddon and L. Saraf, J. Phys. Chem. B, 2004, 108, 19976-19981.

47 S.-R. Jang, R. Vittal and K.-J. Kim, Langmuir, 2004, 20, 9807-9810.

48 Y.-L. Zhao, L. Hu, G. Grüner and J. F. Stoddart, J. Am. Chem. Soc., 2008, 130, 16996-17003.

49 J. Park, P. Deria and M. J. Therien, J. Am. Chem. Soc., 2011, 133, 17156-17159.

50 M. Soler and J. K. McCusker, J. Am. Chem. Soc., 2008, 130, 4708-4724.

51 K. B. Yoon, Chem. Rev., 1993, 93, 321-339.

52 W. J. Youngblood, S.-H. A. Lee, K. Maeda and T. E. Mallouk, Acc. Chem. Res., 2009, 42, 1966-1973.

53 M. Hissler, W. B. Connick, D. K. Geiger, J. E. McGarrah, D. Lipa, R. J. Lachicotte and R. Eisenberg, Inorg. Chem., 2000, 39, 447-457.

54 K. Huang and A. A. Martí, Anal. Chem., 2012, 84, 8075-8082. 55 P.-L. E. Chu, L.-Y. Wang, S. Khatua, A. B. Kolomeisky, S. Link and J. M. Tour, ACS Nano, 2012, 7, 35-41.

56 I. W. Chiang, B. E. Brinson, A. Y. Huang, P. A. Willis, M. J. Bronikowski, J. L. Margrave, R. E. Smalley and R. H. Hauge, J. Phys. Chem. B, 2001, 105, 8297-8301. 